\title{
ANÁLISE DO MERCADO DO ABACAXI COMERCIALIZADO NA CEAGESP - SÃO PAULO ${ }^{1}$
}

\author{
FÁBIO JOSÉ BENGOZI², ALOÍSIO COSTA SAMPAIO³ ${ }^{3}$ ANITA DIAS DE SOUZA GUTIERREZ ${ }^{4}$, \\ VANESSA MARIA RODRIGUES ${ }^{5}$, MARIA LUCIA PALLAMIN ${ }^{6}$
}

RESUMO - A disponibilidade de informações relativas à oferta, preços e sazonalidade do abacaxi é um fator de interesse tanto para produtores quanto para atacadistas, pois podem contribuir para melhor planejamento da época de colheita e comercialização. Dessa maneira, o presente trabalho buscou conhecer a sazonalidade do preço e da quantidade do abacaxi comercializado na CEAGESP - SP, no período de setembro de 2005 a março de 2006. Foram obtidos através de entrevistas aos atacadistas, dados referentes à origem de cada cultivar em estudo, o preço pago ao produtor, o preço de venda praticado pelos atacadistas e a quantidade de frutos recebida no dia. Com o objetivo de compreender o processo de comercialização das cultivares de abacaxi 'Smooth Cayenne' e 'Pérola', foram aplicados questionários aos vendedores de três empresas atacadistas. Através das informações obtidas, sugere-se aos produtores de abacaxi ‘Smooth Cayenne' o escalonamento da produção no período de novembro a fevereiro, preferencialmente de maneira associativista. Os três atacadistas de abacaxi entrevistados vislumbram uma estabilidade na quantidade comercializada de abacaxi na CEAGESP, com previsão de crescimento para a cultivar 'Pérola' e redução para a cultivar 'Smooth Cayenne'. No período de 12 de setembro de 2005 a 20 de março de 2006, observou-se uma estabilidade de preços pagos ao produtor para ambas as cultivares, com elevação de preços entre os dias 23 de janeiro a 06 de março de 2006 para a cultivar 'Pérola' e entre os dias 06 de fevereiro a 06 de março de 2006 para a cultivar 'Smooth Cayenne'. Os atacadistas consideram a cultivar 'Smooth Cayenne' mais valorizada nos meses de novembro a janeiro e a cultivar 'Pérola' de março a junho e de novembro a dezembro. Pelo cenário de estabilização do mercado de abacaxi, torna-se fundamental a garantia de sabor monitorada pelas associações de produtores em parceria com o Centro de Qualidade em Horticultura $(\mathrm{CQH}-$ CEAGESP) e atacadistas, com destaque para a cultivar 'Smooth Cayenne'.

Termos para indexação: Ananas comosus, 'Smooth Cayenne', 'Pérola, 'preços e quantidades'.

\section{ANALYSIS OF THE MARKET OF PINEAPPLE COMMERCIALIZED IN THE CEAGESP-SÃO PAULO}

\begin{abstract}
The information source related to the offer, prices and seasonality of the pineapple fruit is a factor of great interest as to the producers as to the wholesalers. Such information may contribute to a better harvest, commercialization and price evaluation planning of the period. The purpose of the present study was to evaluate the price seasonality and the amount of pineapple commercialized at the Entreposto Terminal de São Paulo (ETSP) of the Companhia de Entrepostos e Armazéns Gerais de São Paulo (CEAGESP) from September/2005 to March/2006. Interviews with wholesalers provided us data concerning to the origin of each variety studied, the price paid to producers, the wholesaler's price and the amount of fruit received each day. In order to understand the process of commercialization of the varieties of 'Smooth Cayenne' and 'Pérola', salespeople employed by the three wholesale companies were asked to answer questionnaires concerning the whole process. Based on marketing information provided by wholesalers, it was suggested that the producers of 'Smooth Cayenne' should organize the production throughout the period of November and February, preferably in the form of an association. The three pineapple wholesalers interviewed predict stability in the amount of pineapple commercialized at the CEAGESP, with an increase in 'Pérola' variety and a decrease in 'Smooth Cayenne' variety. From September $12^{\text {th }}, 2005$ to March $20^{\text {th }}$, 2006, there was a good stability of prices paid to producers for both varieties, with an increase in 'Pérola' prices from January $23^{\text {rd }}$ to March $6^{\text {th }}, 2006$ and in 'Smooth Cayenne' prices from February $6^{\text {th }}$ to March $6^{\text {th }}, 2006$. The wholesalers consider the variety 'Smooth Cayenne' better valued from November to January and the variety 'Pérola' better valued from March to June and in November and December. With the present situation and future predictions of a stable commercialization of the fruit, it is fundamental that its taste is guaranteed and is always monitored by associations of producers in partnerships with the Horticulture Quality Center (CQH-CEAGESP) and with wholesalers as well, particularly for the variety 'Smooth Cayenne'.

Index terms: Ananas comosus, 'Smooth Cayenne', 'Pérola', price and amount.

\footnotetext{
${ }^{1}$ (Trabalho 180-06). Recebido em : 09-11-2006. Aceito para publicação em : 03-08-2007. Parte da Dissertação do primeiro autor apresentada à FCA/ UNESP-Botucatu par a obtenção do título de Mestre.

${ }^{2}$ Engenheiro Agrônomo, MsC. em Horticultura pela FCA/UNESP-Botucatu. E-mail: fjbengozi@fca.unesp.br

${ }^{3}$ Engenheiro Agrônomo, Prof. Dr. do Depto Ciências Biológicas - FC/UNESP-Bauru. E-mail: aloisio@fc.unesp.br

${ }^{4}$ Engenheira Agrônoma, Dra ${ }^{\mathrm{a}}$ do CQH/CEAGESP-SP. E-mail anita@hortibrasil.org.br

${ }^{5}$ Engenheira Agrônoma, Bolsista FAPESP do CQH/CEAGESP-SP. E-mail: vanessa@yahoo.com.br

${ }^{6}$ Bióloga, aluna de Mestrado do Depto de Horticultura da FCA/UNESP-Botucatu. E-mail: pallamin@fca.unesp.br
} 


\section{INTRODUÇÃO}

No Brasil, berço da cultura e um dos maiores produtores mundiais, a cultura é economicamente explorada na maioria dos Estados, dando uma importante contribuição à geração de renda e emprego e, portanto, à fixação do homem no campo (SOUZA e SOUZA, 2000).

No cenário mundial de frutas, o abacaxi ocupou o sétimo lugar em produção, com 15,7 e 15,9 milhões de toneladas em 2004 e 2005, respectivamente, sendo cultivado em mais de 70 países. Segundo dados da FAO (2007), o Brasil figurou, em 2005, como o quarto maior produtor de abacaxi no mundo, com produção de 1,41 milhão de toneladas, sendo superado apenas pela Tailândia (2,05 milhões de toneladas), Filipinas (1,80 milhão de toneladas) e China (1,46 milhão de toneladas).

A produção brasileira de abacaxi está distribuída principalmente nas regiões Nordeste $(40,2 \%)$, Sudeste $(28,92 \%)$ e Norte (26,14\%). O Estado de São Paulo aparece como o sexto maior produtor nacional, representando $5,9 \%$ da produção nacional. O Estado do Pará, com participação de $21,4 \%$ da produção nacional de abacaxi, destacou-se como o maior produtor brasileiro em 2004, seguido dos Estados da Paraíba (18\%), Minas Gerais (15\%), Bahia (8\%) e Rio Grande do Norte (7\%) (IBGE, 2007). Com relação à comercialização do abacaxi na maior Central Atacadista da América Latina (Ceagesp-São Paulo), dados estatísticos tabulados pelo Centro de Qualidade em Horticultura (CQH) demonstraram para a cv. 'Smooth Cayenne', que no período de 2001 a 2005, em setembro e fevereiro, ocorreram, respectivamente, o menor e o maior preço. Para a cv. 'Pérola', os meses de pior e melhor preço foram outubro e março. No aspecto de quantidade, observou-se que a cv. 'Pérola' superou a cv. Hawaí nos anos de 2003 e 2004, restaurando um equilíbrio de volume comercializado no ano de 2005 (CQH - CEAGESP, 2006 Comunicação pessoal).

A disponibilidade de informações relativas à sazonalidade da oferta e de preços do abacaxi é um fator de grande interesse tanto para produtores quanto para atacadistas e comerciantes, pois podem contribuir para um melhor planejamento da época de colheita e comercialização (ALVES et al., 1998). O presente trabalho buscou conhecer a sazonalidade do preço e da quantidade do abacaxi comercializado na CEAGESP - São Paulo, no período de setembro de 2005 a março de 2006. Os dados da qualidade físico-química dos frutos do abacaxi avaliados durante a coleta dos dados serão apresentados em outro artigo.

\section{MATERIAL E MÉTODOS}

O presente trabalho foi desenvolvido no Entreposto Terminal de São Paulo (ETSP) da Companhia de Entrepostos e Armazéns Gerais de São Paulo (CEAGESP) de 12 de setembro de 2005 a 20 de março de 2006. A coleta de dados foi realizada em intervalos de duas semanas, às segundas-feiras (dia de intensa comercialização no mercado) através de entrevistas junto aos atacadistas, registrando dados referentes à origem de cada cultivar em estudo, o preço pago ao produtor, o preço de venda praticado pelos atacadistas e a quantidade de frutos recebida no dia. Os preços pagos ao produtor e de venda dos atacadistas foram obtidos para frutos do tipo 10 , onde o tipo corresponde ao número de frutos acondicionados em uma caixa de comercialização. Os atacadistas informaram os preços pagos ao produtor e de atacado da cultivar 'Pérola' em R $\$$ /unidade que foram transformados para $\mathrm{R} \$ / \mathrm{kg}$. Para os frutos da cultivar 'Smooth Cayenne', o preço pago ao produtor já foi informado pelos atacadistas em $\mathrm{R} \$ / \mathrm{kg}$, e o preço de atacado foi informado em $\mathrm{R} \$$ /caixa e, posteriormente, transformado em $\mathrm{R} \$ / \mathrm{kg}$. Os atacadistas forneceram as informações referentes às quantidades comercializadas em quilogramas para a cultivar 'Smooth Cayenne' e em número de frutos para a cultivar 'Pérola', portanto foi necessária a transformação dessa última para quilogramas. Para essas transformações, foi usado como base o peso médio do fruto coletado no dia para cada cultivar acondicionada em caixas tipo 10 (peso médio ao redor de 1,8 - 2,0 $\mathrm{kg})$

Com o objetivo de compreender o processo de comercialização das cultivares de abacaxi 'Smooth Cayenne' e 'Pérola', foram aplicados questionários a vendedores de três tradicionais empresas atacadistas indicadas pela equipe técnica do CQH/Ceagesp. A aplicação do questionário seguiu a metodologia recomendada por Mattar (1999) e utilizada por Andreucetti et al. (2005) e Almeida (2006) e em levantamento realizado no ETSP da CEAGESP com atacadistas de tomates e pêssegos, respectivamente.

\section{RESULTADOS E DISCUSSÃO}

Os frutos de abacaxi foram provenientes de várias regiões produtoras do País, sendo que frutos da cultivar 'Smooth Cayenne' vieram de Canápolis, em Minas Gerais; e de Bauru e Guaraçaí, em São Paulo, enquanto os frutos da cultivar 'Pérola' procediam de Sapé e Santa Rita, na Paraíba; Miracema do Tocantins, em Tocantins; São Francisco e Campos dos Goytacazes, no Rio de Janeiro; Frutal, em Minas Gerais; Itaberaba, na Bahia; Floresta e Conceição do Araguaia, no Pará; Tuntum, no Maranhão; e Jaraguá, em Goiás.

Analisando-se as Figuras 1 e 2, observa-se que, de 12 de setembro de 2005 a 20 de março de 2006, tanto para os frutos (Tipo 10) da cultivar 'Smooth Cayenne' como para os da cultivar 'Pérola', o comportamento dos preços pagos aos produtores foram semelhantes aos dos preços no atacado, ou seja, de uma forma geral a margem de lucro dos três atacadistas permaneceu sem grandes variações durante o período analisado. No período de 07 de novembro de 2005 (semana 8) a 23 de janeiro de 2006 (semana 19), verifica-se uma grande variação na quantidade de abacaxi 'Smooth Cayenne' comercializado pelos atacadistas. A irregularidade na oferta é prejudicial à cadeia do abacaxi, principalmente aos produtores que precisam comercializar um produto perecível. Nesse sentido, a organização dos produtores, visando ao planejamento para induções florais com produtos à base de ethephon nas diferentes regiões produtoras (Bauru, Guaraçaí e Canápolis), pode contribuir para uma melhor estabilidade de oferta de frutos e, conseqüentemente, para a obtenção de melhores preços por parte dos produtores. Pode-se 
perceber, também, que, para os frutos da cultivar 'Pérola', houve uma melhor remuneração tanto para os produtores quanto para os atacadistas, com exceção para o preço de atacado nas semanas 6 (24-10-2005), 8 (07-11-2005) e 12 (20-02-2006) quando as duas cultivares tiveram preços semelhantes.

As quantidades, em quilogramas, apresentadas nas Figuras 1 e 2 ,são referentes às quantidades comercializadas por três atacadistas do ETSP da CEAGESP, portanto não condizem com a média da quantidade comercializada no período apresentado nas Figuras 3 e 4, pois essas apresentam a média das quantidades totais comercializadas pelo ETSP - CEAGESP, no período de 2001 a 2005

Através da Tabela 1, verifica-se que, apesar da redução do preço pago ao produtor de 'Smooth Cayenne', no período de 26-09-05 (R \$ 0,50/kg) a 05-12-05 (R \$ 0,42/kg), os preços médios de venda praticados pelos três atacadistas mantiveram-se estáveis em $\mathrm{R} \$ 0,85 / \mathrm{kg}$, o que demonstra a maior necessidade de organização dos produtores em relação à dinâmica do mercado. No dia 19-12-06, observa-se pela Figura e Tabela 1, um aumento do volume comercializado de abacaxi 'Smooth Cayenne', bem como pelo preço pago ao produtor (R $\$ 0,60 / \mathrm{kg}$ ). Analisando-se os dados da Tabela 2, nota-se um aumento significativo nos preços médios anuais empregados pelos atacadistas analisados para ambas as cultivares, no período de 2001 a 2005. Com relação à sazonalidade dos preços ao longo do ano, tem-se para a cv. 'Smooth Cayenne' maiores valores nos meses de fevereiro a abril e para a cv. 'Pérola' nos meses de janeiro a março. Ainda através da Tabela 2, observa-se uma boa estabilidade de preços de venda dos atacadistas para a cv. 'Pérola' durante todos os meses de 2005, com exceção para mês de janeiro.

As Figuras 3 e 4 mostram a evolução da quantidade (em toneladas) e do preço de atacado $(\mathrm{R} \$ \mathrm{~kg})$ de abacaxi das cultivares 'Smooth Cayenne' e 'Pérola', respectivamente, nos últimos 5 anos, ou seja, no período de 2001 a 2005 (CQH - CEAGESP, 2006). Os preços estão corrigidos pelo Índice de Preço ao Consumidor (IPC - FIPE), empregando-se a data-base do ano de 2005 para cada mês. O processo de "inflacionar" ou "deflacionar" uma série de pagamentos/recebimentos (valor nominal) para uma determinada data de referência (valor real), traduz em si uma comparação entre as evoluções dos valores monetários em análise e o comportamento dos preços dos produtos enfeixados no índice escolhido (SAMANEZ, 2006). No qüinqüênio 2001-2005, o período de menor oferta (entressafra) para as cultivares deu-se de maio a agosto. Observa-se também que os valores médios de preço no atacado de 2001 a 2005 de janeiro a dezembro são sempre superiores para a cultivar 'Pérola'. Em relação à quantidade comercializada, em toneladas, a cultivar 'Smooth Cayenne' apresentou um valor superior de dezembro a fevereiro e em julho. A Figura 5 mostra uma tendência de redução na quantidade anualmente comercializada do abacaxi 'Smooth Cayenne' em detrimento ao 'Pérola', no período de 2001 a 2004. Em 2005, as quantidades comercializadas para as duas cultivares foram semelhantes (CQH - CEAGESP, 2006).

A Figura 6 mostra as cultivares de abacaxi comercializadas pelos três atacadistas do ETSP da CEAGESP ao longo do ano. Os três atacadistas comercializam a cultivar 'Pérola' ao longo do ano inteiro, sendo que o atacadista 2 comercializou maior volume de junho a dezembro. Já para a cultivar 'Smooth Cayenne', existe variação no volume dessa fruta ao longo do ano pelos três atacadistas entrevistados, sendo que o atacadista 1 comercializa essa fruta de novembro a janeiro, o atacadista 2 , de junho a março, com maior oferta de setembro a dezembro com destaque para o mês de dezembro e o atacadista 3 comercializa essa cultivar ao longo do ano todo.

Pela Figura 7, podem-se observar os meses em que as cultivares 'Smooth Cayenne' e 'Pérola' são consideradas mais saborosas pelos três atacadistas entrevistados. Os atacadistas 2 e 3 consideram que a cultivar 'Pérola' é saborosa durante todo o ano. Os mesmos atacadistas consideram que a cultivar 'Smooth Cayenne' é mais saborosa de novembro a fevereiro e em novembro, respectivamente. Já o atacadista 1 considera a cultivar 'Smooth Cayenne' com sabor mais agradável no mês de dezembro, e a cultivar 'Perola' de fevereiro a maio e de setembro a novembro.

Na Figura 8, são apresentados os meses em que as cultivares 'Smooth Cayenne' e 'Pérola' são consideradas mais valorizadas pelos atacadistas. Para o atacadista 1 , a cultivar 'Smooth Cayenne' é mais valorizada em novembro e dezembro, a 'Pérola' de março a junho devido à baixa oferta da fruta nessa época. $\mathrm{O}$ atacadista 2 considera que a cultivar 'Pérola' alcança bons valores durante todo o ano, e a cultivar 'Smooth Cayenne' de novembro a fevereiro, período em que a cultivar é considerada bastante saborosa. $\mathrm{O}$ atacadista 3 considera novembro e dezembro como os meses de maior valorização para as duas cultivares.

Esses resultados indicam, de maneira clara, aos produtores de abacaxi 'Smooth Cayenne' dos Estados de São Paulo e Minas Gerais a importância de se planejar o escalonamento da produção de novembro a fevereiro, através das técnicas de manejo cultural e, principalmente, respeitando-se o ponto de colheita adequado, ou seja, com monitoramento por amostragem do teor de sólidos solúveis, cujo valor mínimo sugerido pelo $\mathrm{CQH} /$ Ceagesp seria de 12 graus brix. Esse método tem sido adotado no programa 'Garantia de Sabor' da Ceagesp em parceria com associações de fruticultores, para outras frutas não-climatéricas como as uvas finas, com bons resultados.

Os três atacadistas declararam realizar as compras através de preço feito, ou seja, o abacaxi vem da lavoura com preço estabelecido, e o pagamento é feito normalmente com prazo de 30 dias. Aproximadamente $50 \%$ das compras são realizadas pessoalmente, ou seja, através de compradores nas propriedades, e os outros $50 \%$ através do telefone. Apenas o atacadista 2 realiza toda sua aquisição de frutas pessoalmente. Para os dois atacadistas que comercializam as duas cultivares, cerca de $70 \%$ da importância da comercialização em quantidade é proveniente da cultivar 'Pérola', e 30\% da cultivar 'Smooth Cayenne'. Já para o atacadista 1, praticamente a totalidade da quantidade comercializada é da cultivar 'Pérola'.

A previsão da evolução da quantidade de comercialização pode ser observada na Tabela 3 , onde dois atacadistas consideram que a quantidade de abacaxi das duas cultivares era maior há 5 anos, ou seja, ocorreu um decréscimo nas vendas da fruta, sendo que a previsão é de estabilização na comercialização da cultivar 'Pérola' e redução da cultivar 'Smooth Cayenne' para os próximos 
5 anos. Apenas o atacadista 2 considera que houve inversão para as duas cultivares, sendo que houve redução na quantidade comercializada de 'Smooth Cayenne' nos últimos 5 anos, com uma previsão de aumento de comercialização da cultivar 'Perola'. Pelo cenário de estabilização do mercado de abacaxi, torna-se fundamental a garantia de sabor monitorada pelos produtores individualmente ou associações em parceria com o Centro de Qualidade em Horticultura (CQH - CEAGESP) e atacadistas, principalmente para a 'Smooth Cayenne', que apresenta uma acidez natural mais elevada quando comparada com a cv. 'Pérola'. Nesse programa, há o levantamento do brix dos frutos coletados por amostragem através de refratômetro manual na origem (local de produção) e no destino (atacadista do Ceagesp-São Paulo), com acompanhamento dos resultados pela equipe técnica do $\mathrm{CQH}$.

Segundo os três atacadistas entrevistados, aproximadamente $75 \%$ do total de abacaxi, das duas cultivares são vendidos para a Grande São Paulo e $25 \%$ para o Interior e Litoral do Estado de São Paulo.

$\mathrm{Na}$ opinião dos atacadistas para que haja aumento no volume comercializado do abacaxi o produtor deve escalonar melhor a safra para que haja um equilíbrio maior na oferta da fruta durante o ano, e procurar aumentar o consumo através da melhoria da qualidade dos frutos.

TABELA 1 - Preços médios pagos ao produtor e de venda dos atacadistas das cultivares 'Smooth Cayenne' e 'Pérola' comercializados por três atacadistas na CEAGESP - SP, de 12 de setembro de 2005 a 20 de março de 2006.

\begin{tabular}{|c|c|c|c|c|c|c|c|c|}
\hline \multirow{3}{*}{ DATA } & \multicolumn{4}{|c|}{ cv. Smooth Cayenne (Havaí)* } & \multicolumn{4}{|c|}{ cv. Pérola** } \\
\hline & \multicolumn{2}{|c|}{ Pago ao Produtor } & \multicolumn{2}{|c|}{ Pago ao Atacadista } & \multicolumn{2}{|c|}{ Pago ao Produtor } & \multicolumn{2}{|c|}{ Pago ao Atacadista } \\
\hline & Preço/fruto & Preço/kg & Preço/fruto & Preço/kg & Preço/fruto & Preço/kg & Preço/fruto & Preço/kg \\
\hline $12-09-05$ & $\mathrm{R} \$ 1,10$ & $\mathrm{R} \$ 0,50$ & $\mathrm{R} \$ 1,50$ & $\mathrm{R} \$ 0,68$ & R\$ 1,43 & R\$ 0,79 & $\mathrm{R} \$ 2,50$ & R\$ 1,39 \\
\hline $26-09-05$ & $\mathrm{R} \$ 1,10$ & $\mathrm{R} \$ 0,50$ & $\mathrm{R} \$ 1,88$ & $\mathrm{R} \$ 0,85$ & $\mathrm{R} \$ 1,15$ & $\mathrm{R} \$ 0,64$ & $\mathrm{R} \$ 2,10$ & $\mathrm{R} \$ 1,17$ \\
\hline $10-10-05$ & $\mathrm{R} \$ 1,06$ & $\mathrm{R} \$ 0,48$ & $\mathrm{R} \$ 1,88$ & $\mathrm{R} \$ 0,85$ & $\mathrm{R} \$ 1,33$ & $\mathrm{R} \$ 0,74$ & $\mathrm{R} \$ 2,30$ & $\mathrm{R} \$ 1,28$ \\
\hline $24-10-05$ & $\mathrm{R} \$ 1,03$ & $\mathrm{R} \$ 0,47$ & $\mathrm{R} \$ 1,88$ & $\mathrm{R} \$ 0,85$ & $\mathrm{R} \$ 1,18$ & $\mathrm{R} \$ 0,66$ & $\mathrm{R} \$ 1,83$ & $\mathrm{R} \$ 1,02$ \\
\hline $07-11-05$ & $\mathrm{R} \$ 1,21$ & $\mathrm{R} \$ 0,55$ & R\$ 1,88 & $\mathrm{R} \$ 0,85$ & $\mathrm{R} \$ 1,30$ & $\mathrm{R} \$ 0,72$ & $\mathrm{R} \$ 1,83$ & $\mathrm{R} \$ 1,02$ \\
\hline $28-11-05$ & $\mathrm{R} \$ 0,99$ & $\mathrm{R} \$ 0,45$ & $\mathrm{R} \$ 1,88$ & $\mathrm{R} \$ 0,85$ & $\mathrm{R} \$ 1,20$ & $\mathrm{R} \$ 0,67$ & $\mathrm{R} \$ 2,10$ & $\mathrm{R} \$ 1,17$ \\
\hline $05-12-05$ & $\mathrm{R} \$ 0,92$ & $\mathrm{R} \$ 0,42$ & $\mathrm{R} \$ 1,88$ & $\mathrm{R} \$ 0,85$ & $\mathrm{R} \$ 1,30$ & $\mathrm{R} \$ 0,72$ & R\$ 1,98 & $\mathrm{R} \$ 1,10$ \\
\hline $19-12-05$ & $\mathrm{R} \$ 1,32$ & $\mathrm{R} \$ 0,60$ & $\mathrm{R} \$ 2,00$ & $\mathrm{R} \$ 0,91$ & $\mathrm{R} \$ 1,23$ & $\mathrm{R} \$ 0,68$ & $\mathrm{R} \$ 2,10$ & $\mathrm{R} \$ 1,17$ \\
\hline 09-01-06 & $\mathrm{R} \$ 1,17$ & $\mathrm{R} \$ 0,53$ & $\mathrm{R} \$ 2,50$ & $\mathrm{R} \$ 1,14$ & $\mathrm{R} \$ 1,00$ & $\mathrm{R} \$ 0,71$ & R\$ 1,84 & $\mathrm{R} \$ 1,31$ \\
\hline $23-01-06$ & RS 1,54 & R\$ 0,70 & $\mathrm{R} \$ 2,50$ & $\mathrm{R} \$ 1,14$ & R\$ 2,50 & RS 1,39 & R\$ 3,94 & $\mathrm{R} \$ 2,19$ \\
\hline $06-02-06$ & R\$ 1,98 & R\$ 0,90 & $\mathrm{R} \$ 2,50$ & $\mathrm{R} \$ 1,14$ & R\$ 2,60 & RS 1,44 & $\mathrm{R} \$ 3,94$ & $\mathrm{R} \$ 2,19$ \\
\hline $20-02-06$ & R\$ 2,42 & RS 1,10 & $\mathrm{R} \$ 3,75$ & $\mathrm{R} \$ 1,70$ & R\$ 2,10 & RS 1,17 & $\mathrm{R} \$ 3,33$ & $\mathrm{R} \$ 1,85$ \\
\hline $06-03-06$ & R\$ 2,20 & R\$ 1,00 & $\mathrm{R} \$ 3,50$ & $\mathrm{R} \$ 1,59$ & R\$ 2,17 & RS 1,21 & $\mathrm{R} \$ 3,59$ & R\$ 1,99 \\
\hline $20-03-06$ & $\mathrm{R} \$ 1,32$ & $\mathrm{R} \$ 0,60$ & $\mathrm{R} \$ 2,50$ & $\mathrm{R} \$ 1,14$ & $\mathrm{R} \$ 1,37$ & $R \$ 0,76$ & $\mathrm{R} \$ 2,40$ & R\$ 1,33 \\
\hline
\end{tabular}

TABELA 2 - Preços médios mensais de atacado das cultivares de abacaxi 'Smooth Cayenne' e 'Pérola, comercializado no ETSP CEAGESP de 2001 a 2005, em reais por quilo (CQH - CEAGESP, 2006).

\begin{tabular}{|c|c|c|c|c|c|c|c|c|c|c|c|c|}
\hline \multirow[t]{2}{*}{ Meses } & \multicolumn{5}{|c|}{ cv. Smooth Cayenne (Havaí) - R\$/kg } & \multirow{2}{*}{$\begin{array}{l}\text { Média } \\
\text { Havaí }\end{array}$} & \multicolumn{5}{|c|}{ cv. Pérola - R\$/kg } & \multirow{2}{*}{$\begin{array}{l}\text { Média } \\
\text { Pérola }\end{array}$} \\
\hline & 2001 & 2002 & 2003 & 2004 & 2005 & & 2001 & 2002 & 2003 & 2004 & 2005 & \\
\hline Janeiro & 0,48 & 0,44 & 0,59 & $\mathbf{1 , 1 5}$ & 0,69 & 0,67 & 1,38 & 1,42 & 1,93 & 3,28 & 1,92 & $\mathbf{1 , 9 9}$ \\
\hline Fevereiro & 0,46 & 0,43 & 0,65 & $\mathbf{1 , 1 1}$ & $\mathbf{1 , 0 6}$ & $\mathbf{0 , 7 4}$ & 1,28 & 1,66 & 1,81 & 2,43 & 2,31 & $\mathbf{1 , 9 0}$ \\
\hline Março & 0,48 & 0,40 & 0,67 & $\mathbf{0 , 8 1}$ & 1,26 & $\mathbf{0 , 7 2}$ & 1,33 & 1,48 & 1,74 & 2,71 & 2,63 & $\mathbf{1 , 9 8}$ \\
\hline Abril & 0,45 & 0,41 & 0,59 & $\mathbf{1 , 0 0}$ & $\mathbf{1 , 0 3}$ & 0,70 & 1,43 & 1,47 & 1,68 & 2,21 & 2,36 & $\mathbf{1 , 8 3}$ \\
\hline Maio & 0,43 & 0,40 & 0,57 & 1,02 & 0,80 & $\mathbf{0 , 6 4}$ & 1,55 & 1,58 & 1,49 & 2,27 & 2,18 & $\mathbf{1 , 8 1}$ \\
\hline Junho & 0,44 & 0,44 & 0,53 & 0,86 & 0,79 & $\mathbf{0 , 6 1}$ & 1,75 & 1,55 & 1,45 & 1,94 & 2,08 & 1,76 \\
\hline Julho & 0,42 & 0,42 & 0,48 & 0,87 & 0,76 & $\mathbf{0 , 5 9}$ & 1,70 & 1,49 & 1,38 & 1,93 & 2,03 & 1,71 \\
\hline Agosto & 0,41 & 0,40 & 0,53 & 0,80 & 0,80 & $\mathbf{0 , 5 9}$ & 1,56 & 1,49 & 1,43 & 1,78 & 2,20 & $\mathbf{1 , 6 9}$ \\
\hline Setembro & 0,41 & 0,38 & 0,56 & 0,69 & 0,76 & 0,56 & 1,55 & 1,28 & 1,51 & 1,50 & 2,36 & 1,64 \\
\hline Outubro & 0,41 & 0,41 & 0,63 & 0,73 & 0,75 & $\mathbf{0 , 5 9}$ & 1,50 & 1,31 & 1,45 & 1,47 & 2,28 & $\mathbf{1 , 6 0}$ \\
\hline Novembro & 0,47 & 0,42 & 0,67 & 0,73 & 0,77 & $\mathbf{0 , 6 1}$ & 1,47 & 1,43 & 1,43 & 1,47 & 2,24 & $\mathbf{1 , 6 1}$ \\
\hline Dezembro & 0,41 & 0,42 & 0,65 & 0,78 & 0,83 & $\mathbf{0 , 6 2}$ & 1,40 & 1,42 & 1,56 & 1,63 & 2,22 & 1,65 \\
\hline $\begin{array}{c}\text { Média } \\
\text { anual } \\
\end{array}$ & $\mathbf{0 , 4 4}$ & $\mathbf{0 , 4 1}$ & $\mathbf{0 , 5 9}$ & $\mathbf{0 , 8 8}$ & $\mathbf{0 , 8 6}$ & & 1,49 & 1,46 & 1,57 & 2,05 & 2,24 & \\
\hline
\end{tabular}

TABELA 3 - Evolução da quantidade dos frutos de abacaxi 'Smooth Cayenne' e 'Pérola' comercializados por três atacadistas, na CEAGESP-SP

\begin{tabular}{lcccccc}
\hline & \multicolumn{2}{c}{ Atacadista 1 } & \multicolumn{2}{c}{ Atacadista 2 } & \multicolumn{2}{c}{ Atacadista 3 } \\
\cline { 2 - 6 } $\begin{array}{c}\text { Histórico e } \\
\text { Previsão }\end{array}$ & $\begin{array}{c}\text { 'Smooth } \\
\text { Cayenne' }\end{array}$ & 'Pérola' & $\begin{array}{c}\text { 'Smooth } \\
\text { Cayenne' }\end{array}$ & 'Pérola' & $\begin{array}{c}\text { 'Smooth } \\
\text { Cayenne' }\end{array}$ & 'Pérola' \\
\hline 5 Anos Atrás & maior & maior & maior & menor & maior & maior \\
\hline Daqui 5 Anos & menor & igual & menor & maior & menor & igual \\
\hline
\end{tabular}




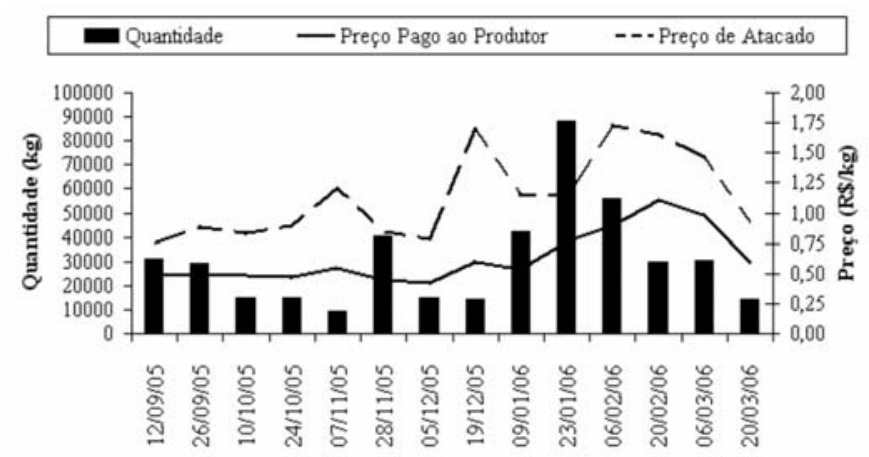

FIGURA 1- Quantidade (kg) e preços pago ao produtor e de atacado ( $\mathrm{R} \$ / \mathrm{kg})$ do abacaxi 'Smooth Cayenne' comercializado por três atacadistas na CEAGESP - SP, de 12 de setembro de 2005 a 20 de março de 2006.

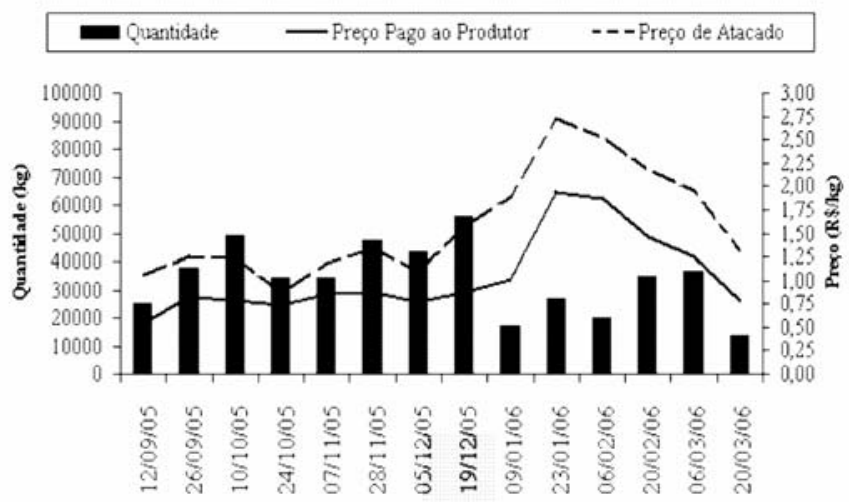

FIGURA 2 - Quantidade (kg) e preços pago ao produtor e de atacado $(\mathrm{R} \$ / \mathrm{kg})$ do abacaxi 'Pérola' comercializado por três atacadistas na CEAGESP - SP, de 12 de setembro de 2005 a 20 de março de 2006.

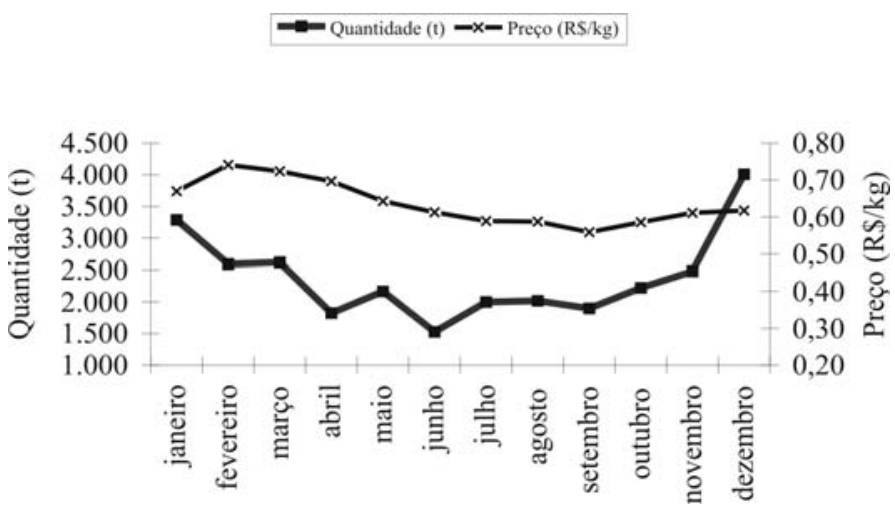

FIGURA 3 - Quantidade média média mensal, em toneladas, e preço de atacado atualizado mensalmente para o ano de 2005 (IPC-FIPE), em R \$/kg abacaxi 'Smooth Cayenne' comercializado no ETSP - CEAGESP de 2001 a 2005 (CQH - CEAGESP, 2006).

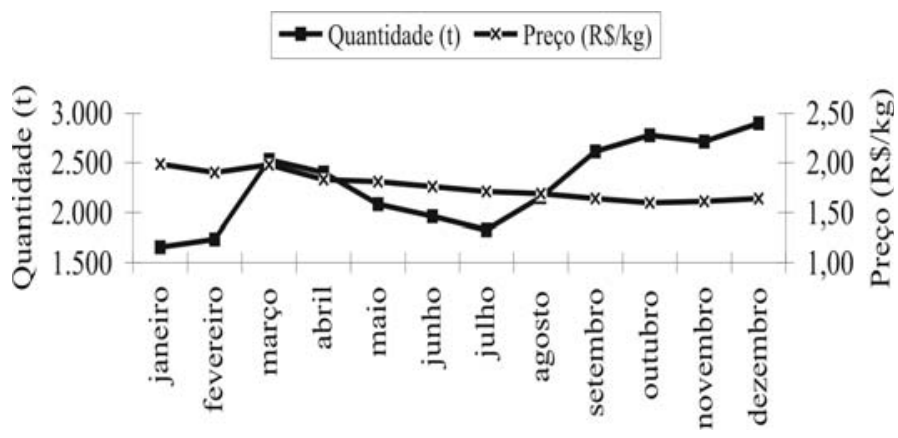

FIGURA 4 - Quantidade média mensal, em toneladas, e preço de atacado atualizado mensalmente para o ano de 2005 (IPC-FIPE), em R $\$ / \mathrm{kg}$ abacaxi 'Pérola' comercializado no ETSP - CEAGESP de 2001 a 2005 (CQH-CEAGESP, 2006).

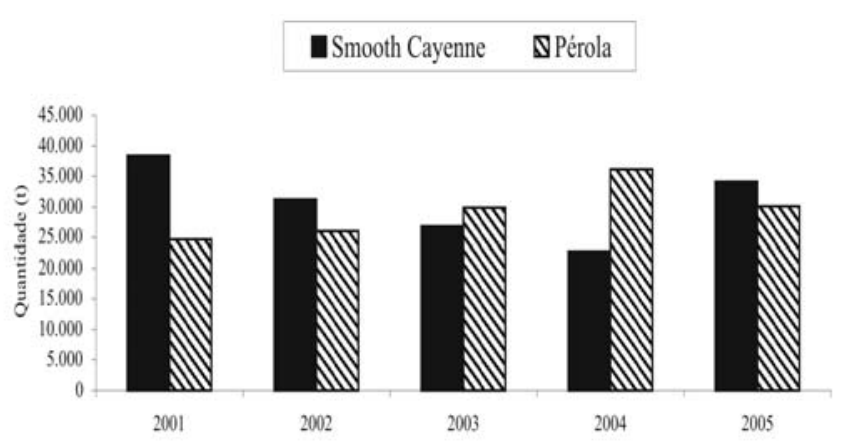

FIGURA 5 -Quantidades totais anuais, em toneladas, dos abacaxis 'Smooth Cayenne' e 'Pérola' comercializados no ETSP - CEAGESP no período de 2001 a 2005 (CQH -CEAGESP, 2006).

* Meses de maior oferta da fruta.

** Pico de oferta da fruta.

FIGURA 6 - Cultivares de abacaxi comercializadas ao longo do ano por três atacadistas. 


\begin{tabular}{|c|c|c|c|c|c|c|c|c|c|c|c|c|c|}
\hline & Cultivar & jan & fer & mar & abr & $\mathrm{mai}$ & jw & jul & 200 & set & out & nor & dez \\
\hline Atacadista & 'Smooth Cyenne' & & & & & & & & & & & & \\
\hline 1 & Perola' & & & & & & & & & & & & \\
\hline Atacadista & 'SmoothCogenne' & & & & & & & & & & & & \\
\hline 2 & Pérola' & & & & & & & & & & & & \\
\hline Atacadista & 'SnoothCegenne' & & & & & & & & & & & & \\
\hline 3 & Pefrola' & & & & & & & & & & & & \\
\hline
\end{tabular}

FIGURA 7 - Meses em que as cultivares de abacaxi 'Smooth Cayenne' e 'Pérola' são consideradas mais saborosas pelos três atacadistas

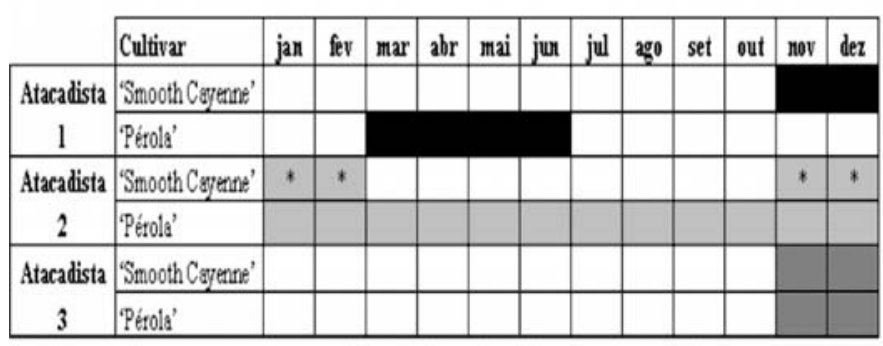

* Meses de maior oferta da fruta.

FIGURA 8 - Meses em que as cultivares de abacaxi 'Smooth Cayenne' e 'Pérola' são consideradas mais valorizadas pelos três atacadistas.

\section{CONCLUSÕES}

1 - Através das informações fornecidas pelos três atacadistas em relação ao mercado, sugere-se aos produtores de abacaxi 'Smooth Cayenne' o escalonamento total da produção no período de novembro a fevereiro.

2 - Os três atacadistas de abacaxi entrevistados vislumbram uma estabilidade na quantidade comercializada de abacaxi na CEAGESP, com previsão de crescimento para a cultivar 'Pérola' e redução para a cultivar 'Smooth Cayenne', decorrente das diferenças naturais de acidez entre os dois materiais genéticos.

3- No período de 12 de setembro de 2005 a 20 de março de 2006, observou-se uma boa estabilidade de preços pagos ao produtor para ambas as cultivares, com pequena elevação de preços entre os dias 23 de janeiro e 06 de março de 2006 para a cultivar 'Pérola', e entre os dias 06 de fevereiro e 06 de março de 2006 para a cultivar 'Smooth Cayenne'.

4 - Os atacadistas consideram a cultivar 'Smooth Cayenne' mais valorizada nos meses de novembro a janeiro, e a cultivar 'Pérola', de março a junho e de novembro a dezembro.

\section{REFERÊNCIAS}

ALMEIDA, G. V. B. de. Características qualitativas de pêssegos produzidos em Paranapanema-SP, safra 2005, e sua valoração no mercado atacadista de São Paulo. 2006. 66 f. Dissertação (Mestrado em Agronomia/Produção Vegetal) - Faculdade de Ciências Agrárias e Veterinárias, Universidade Estadual Paulista, Jaboticabal, 2006.

ALVES, A. de. A. et al. Manejo e avaliação da soca de abacaxi 'Pérola' nas condições do semi-árido de Itaberaba, Bahia. Revista Brasileira de Fruticultura, Jaboticabal, v. 20, n. 3, p. 265-270, 1998.

ANDREUCETTI, C. et al. Caracterização da comercialização de tomate de mesa na CEAGESP: perfil dos atacadistas. Horticultura Brasileira, Campinas, v.23, n.2, p.328-333, 2005.

CQH - Centro de Qualidade em Horticultura. São Paulo: CEAGESP, 2006.

FAO - Food and Agriculture Organization. FAOSTAT Database. Disponível em: <http://faostat.fao.org/faostat. Acesso em: 04 abr.2006.

IBGE - Instituto Brasileiro de Geografia e Estatística. Disponível em: $<$ http//www.sidra.ibge.gov.br.>. Acesso em: 04 abr.2006.

MATTAR, F. N. Pesquisa de Marketing: metodologia, planejamento. 5.ed. São Paulo: Atlas, 1999. 339 p.

SAMANEZ, C.P. Matemática financeira: aplicações à análise de investimentos. 4.ed. São Paulo: Prentice-Hall, 2006. 273 p.

SOUZA, J. da. S.; SOUZA, L. F. da. S. Aspectos econômicos. In: REINDHARDT, D. H., SOUZA, L. F. da. S.; CABRAL, J. R. S. (Org.). Abacaxi. Produção: aspectos técnicos. Cruz das Almas: Embrapa Mandioca e Fruticultura; Brasília, DF: EMBRAPA Comunicação para Transferência de Tecnologia, 2000. p. 10. (Frutas do Brasil, 7). 